\title{
Dynamical mass estimates for two luminous star clusters in galactic merger remnants ${ }^{\star}$
}

\author{
N. Bastian ${ }^{1,2}$, R. P. Saglia ${ }^{3}$, P. Goudfrooij ${ }^{4}$, M. Kissler-Patig ${ }^{2}$, C. Maraston ${ }^{5}$, F. Schweizer ${ }^{6}$, and M. Zoccali ${ }^{7}$ \\ 1 Department of Physics and Astronomy, University College London, Gower Street, London, WC1E 6BT, UK \\ e-mail: bastian@star.ucl.ac.uk \\ 2 European Southern Observatory, Karl-Schwarzschild-Strasse 2, 85748 Garching b. München, Germany \\ 3 Max-Planck-Institut für Extraterrestrische Physik, Giessenbachstrasse, 85748 Garching, Germany \\ 4 Space Telescope Science Institute, 3700 San Martin Drive, Baltimore, MD 21218, USA \\ 5 University of Oxford, Denys Wilkinson Building, Keble Road, Oxford, OX13RH, UK \\ ${ }^{6}$ Carnegie Observatories, 813 Santa Barbara Str., Pasadena, CA 91101-1292, USA \\ 7 Pontificia Universidad Católica de Chile, Departamento de Astronomía y Astrofísica, Av. Vicuña Mackenna 4860, \\ 782-0436 Macul, Santiago, Chile
}

Received 8 September 2005 / Accepted 28 October 2005

ABSTRACT

We present high-dispersion spectra of two extremely massive star clusters in galactic merger remnants, obtained using the UVES spectrograph mounted on the ESO Very Large Telescope. One cluster, W30, is located in the $\sim 500 \mathrm{Myr}$ old merger remnant NGC 7252 and has a velocity dispersion and effective radius of $\sigma=27.5 \pm 2.5 \mathrm{~km} \mathrm{~s}^{-1}$ and $R_{\mathrm{eff}}=9.3 \pm 1.7 \mathrm{pc}$, respectively. The other cluster, G114, located in the $\sim 3 \mathrm{Gyr}$ old merger remnant NGC 1316, is much more compact, $R_{\text {eff }}=4.08 \pm 0.55 \mathrm{pc}$, and has a velocity dispersion of $\sigma=42.1 \pm 2.8 \mathrm{~km} \mathrm{~s}{ }^{-1}$. These measurements allow an estimate of the virial mass of the two clusters, yielding $\mathcal{M}_{\mathrm{dyn}}(W 30)=1.59( \pm 0.26) \times 10^{7} \mathcal{M}_{\odot}$ and $\mathcal{M}_{\mathrm{dyn}}(G 114)=$ $1.64( \pm 0.13) \times 10^{7} \mathcal{M}_{\odot}$. Both clusters are extremely massive, being more than three times heavier than the most massive globular clusters in the Galaxy. For both clusters we measure light-to-mass ratios, which when compared to simple stellar population (SSP) models of the appropriate age, are consistent with a Kroupa-type stellar mass function. Using measurements from the literature we find a strong age dependence on how well SSP models (with underlying Kroupa or Salpeter-type stellar mass functions) fit the light-to-mass ratio of clusters. Based on this result we suggest that the large scatter in the light-to-mass ratio of the youngest clusters is not due to variations in the underlying stellar mass function, but instead to the rapidly changing internal dynamics of young clusters. Based on sampling statistics we argue that while W30 and G114 are extremely massive, they are consistent with being the most massive clusters formed in a continuous power-law cluster mass distribution. Finally, based on the positions of old globular clusters, young massive clusters (YMCs), ultra-compact dwarf galaxies (UCDs) and dwarf-globular transition objects (DGTOs) in $\kappa$-space we conclude that 1) UCDs and DGTOs are consistent with the high mass end of star clusters and 2) YMCs occupy a much larger parameter space than old globular clusters, consistent with the idea of preferential disruption of star clusters.

Key words. galaxies: star clusters - galaxies: interactions - galaxies: individual: NGC 1316 - galaxies: individual: NGC 7252

\section{Introduction}

Our concept of star clusters has changed rapidly during the past two decades. The first resolved young clusters with masses comparable to those of the traditional globular clusters (taken with the Hubble Space Telescope Holtzman et al. 1992) confirmed the suggestions of Schweizer (1987) that mergers of galaxies may produce "young" globular cluster sized objects. These results were rapidly followed by the discovery of additional young massive clusters (YMCs) in other galaxy mergers, as well as in dwarf, starburst, and normal galaxies (see reviews

* Based on Observations at the Very Large Telescope of the European Southern Observatory, Paranal/Chile under Program 073.D-0305(B). by Whitmore 2003 and Larsen 2004). Even our own galaxy is producing YMCs with comparable masses and sizes to those observed in merging galaxies, e.g. Westerlund 1 (Clark et al. 2005). The apparent ubiquity of these objects has raised the question of how "universal" their detailed properties are, in particular concerning their formation and subsequent evolution.

In order to address this and other questions regarding YMCs, we have begun a programme to obtain kinematic and structural properties of star clusters which lie at the extreme high-end of the distribution of observed (luminous) masses. Our first result, presented in Maraston et al. (2004) was for the extremely luminous star cluster, W3, in the galactic merger remnant NGC 7252. Combining the velocity dispersion measured with $U V E S$ on the VLT $\left(45 \pm 5 \mathrm{~km} \mathrm{~s}^{-1}\right)$ with the size 
determined from $H S T$ images $\left(R_{\text {eff }}=17.5 \pm 1.8\right)$ led to the dynamical mass estimate of $8( \pm 2) \times 10^{7} \mathcal{M}_{\odot}$. This mass and that estimated from photometric methods (see Maraston et al. 2004, for details) were in excellent agreement, arguing that the stellar mass function within W3 was Salpeter-like.

Using similar techniques as were employed in the above work, some studies have suggested that the stellar mass function in star clusters can vary substantially (e.g. Smith \& Gallagher 2001), while others have reported standard Kroupa (2002) or Salpeter (1955) type stellar mass functions (e.g. Larsen et al. 2004). These discrepant results have left the question of the variance of the stellar mass function of massive YMCs open to debate.

Additionally, detailed knowledge of the internal dynamics and structural parameters of YMCs has allowed a comparison between them and other gravitationally bound systems. In Maraston et al. (2004) we showed that W3 is too diffuse for its mass when compared with old globular clusters, whereas it is too compact relative to dwarf galaxies. However, we also showed that W3's properties were extremely similar to those of massive point-like objects discovered in Fornax (Hilker et al. 1999). Based on this similarity, Bastian et al. (2005b) have suggested a mechanism which may allow massive star clusters to exist far from the main body of the host galaxy, namely the formation of massive clusters in the tidal debris of galactic interactions/mergers.

Following up on these results, we have obtained high resolution $U V E S$ optical spectra of two additional highly luminous star cluster candidates in galactic merger remnants. The first is the cluster W30, the second brightest cluster in NGC 7252. W30 has an estimated age of $\sim 300-500 \mathrm{Myr}$ and a metallicity between half solar and solar, estimated from optical spectra (Schweizer \& Seitzer 1998) as well as from optical and near-infrared photometry (Maraston et al. 2001). The observed magnitude of W30 is $m_{V}(\mathrm{~W} 30)=19.46 \mathrm{mag}$ and it has a $(V-I)$ colour of $0.63 \mathrm{mag}$ (Miller et al. 1997). The second cluster in this study is G114 in NGC 1316. Based on optical and near-infrared photometry along with near-infrared spectroscopy, Goudfrooij et al. (2001a,b) estimate an age of $3.0 \pm$ 0.5 Gyr for G114. This cluster, the brightest one in NGC 1316, has an observed magnitude $m_{B}(\mathrm{G} 114)=19.63 \mathrm{mag}$ and $(B-I)$ colour $1.87 \mathrm{mag}$. Throughout this work we adopt the distances to NGC 7252 and NGC 1316 which were used in Maraston et al. (2004) and Goudfrooij et al. (2001a,b) respectively, namely 64.4 and $22.9 \mathrm{Mpc}$. Figures 1 and 2 show the HST/WFPC2 planetary camera chip images of NGC 7252 and NGC 1316, respectively, along with the slit sizes and positions used. In Fig. 1 we also mark the massive star cluster W3.

The luminosities of G114 and W30 (assuming a Salpeter or Kroupa-type stellar IMF) imply that they have extremely high masses, more than 3 times that of the most massive globular cluster in the Galaxy, $\omega$ Cen which has a mass of $\sim 3 \times 10^{6} \mathcal{M}_{\odot}$ (Meylan \& Mayor 1986).

In this work we investigate the structural and kinematic properties of these two massive star clusters in order to determine how they relate to "normal" young and old globular clusters. In Sect. 2 we measure the effective radii of the two clusters using high-resolution $H S T$ imaging, and in Sect. 3 we

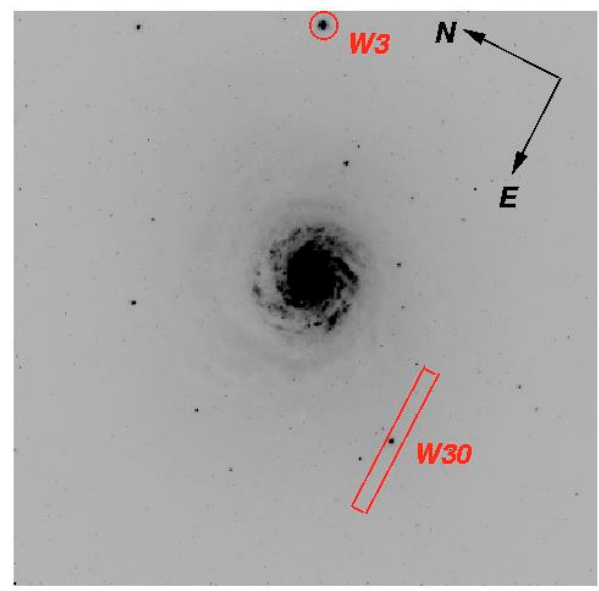

Fig. 1. $F 555 W$ image of the centre of NGC 7252 showing the slit size and position, as well as cluster $\mathrm{W} 3$ for reference. The image is 740 pixels on a side which corresponds to a linear distance of $\sim 10.5 \mathrm{kpc}$ at the assumed distance of $64.4 \mathrm{Mpc}$.

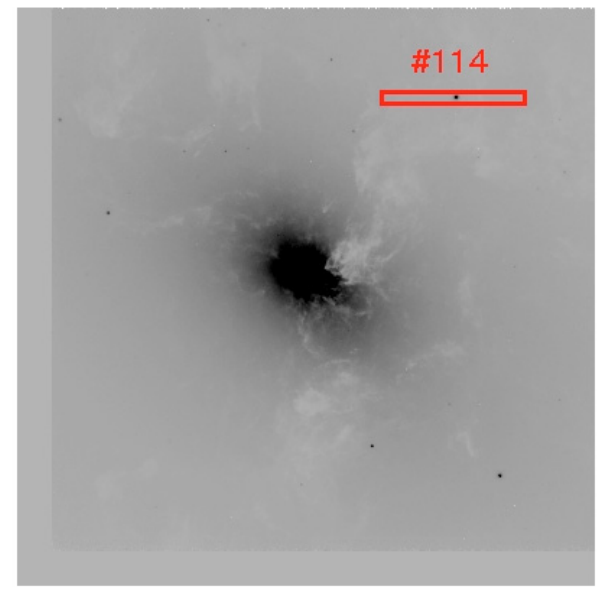

Fig. 2. F450W image of the centre of NGC 1316 showing the slit position. The image is 800 pixels on a side which corresponds to a linear distance of $\sim 4 \mathrm{kpc}$ at the assumed distance of $22.9 \mathrm{Mpc}$. North is down and east is to the right in this image.

determine their velocity dispersions. We combine these results in Sect. 4 to estimate the dynamical mass of the clusters. In Sect. 5 we discuss the implications of our results in terms of the underlying stellar mass function of the clusters, and compare their properties with other bound stellar systems. Finally, we summarise the results and present our conclusions in Sect. 6 .

\section{Determination of the cluster sizes}

Structural parameters for clusters G114 and W30 were measured on Wide Field Planetary Camera 2 (WFPC2) and on Advanced Camera for Surveys (ACS) HST images. The images for W30 are presented in detail in Miller et al. (1997). Here we note that W30 is located on the Planetary Camera chip. For G114 we measured the size on both the WFPC2 (data presented in Goudfrooij et al. 2001b) and ACS (presented in Goudfrooij et al. 2004) images. 
Sizes were found using the ISHAPE routine of Larsen (1999). This routine convolves the PSF with a specified model profile of varying sizes and fits it directly to the images. The outputs of this routine for the best fitting model are the FWHM of the major axis, the minor to major axis ratio, and the goodness of fit. For the WFPC2 images we used a PSF generated by TinyTim (Krist \& Hook 1997) at the exact location of the cluster on the chip. We used the drizzled ACS images to eliminate the geometric distortion of the camera. The PSF for each filter of the ACS images was constructed using sources from ACS observations of the globular cluster 47-Tuc.

For each cluster we fit multiple profiles to the images in each of the observed filters, including King, Moffat, and Gaussian profiles.

\subsection{NGC 1316:G114}

Using the $F 450 W W F P C 2$ observations, we fit eight profiles to the image of the cluster. The results are shown in Table 1. In addition to the set profiles, we also fit a Moffat profile with a variable index. The best fitting model is one with an index 1.76. Using this profile we measure an effective radius of $4.08 \mathrm{pc}$, which is remarkably close to the mean of the size determined using all the other profiles $(4.10 \mathrm{pc})$. We estimate the error on the size as the standard deviation of the size measured for the different profiles, $0.25 \mathrm{pc}$.

We have also measured the size of G114 on $F 555 W$ and F814W HST-ACS images. In Table 1 we show the results for the fits using the best fitting profile from the WFPC2 observations. We find that the size determined on the $F 555 W_{A C S}$ images is $\sim 15 \%$ larger than that found on the WFPC2 images. However the size measured on the $F 814 W_{A C S}$ images is $\sim 15 \%$ smaller than on the WFPC2 images.

As the three images all give approximately the same result we conclude that G114 is resolved, and we assign the size of $R_{\text {eff }}(\mathrm{G} 114)=4.08 \pm 0.55 \mathrm{pc}$.

\subsection{NGC 7252:W30}

Using the $F 555 W W F P C 2$ image of W30, we find that the best fitting model is a King profile, with concentration factor 100 . Using this profile we measure an effective radius of $8.3 \mathrm{pc}$. However the mean size of the other profiles gave $10.3 \mathrm{pc}$. Table 2 gives the determined size of W30 for different profiles. There is a clear decreasing $\chi^{2}$ trend as one goes to smaller radii (i.e. closer to the best fitting radius). However, a King profile with concentration factor 30 along with a Moffat profile with index 1.5 are also acceptable fits in terms of their $\chi^{2}$. We therefore estimate the effective radius of W30 to be $9.3 \pm 1.7 \mathrm{pc}$, where the error of $1.7 \mathrm{pc}$ convers the full range of acceptable profile fits.

\section{Velocity dispersion}

We observed NGC 7252:W30 and NGC 1316:G114 with the UltraViolet Echelle Spectrograph (UVES) mounted on the ESO/VLT on the nights of Sept. 13-16th, 2004. We used the red arm, CD\#3 grating centred on $5200 \AA$. This resulted
Table 1. Effective radius of NGC 1316:G114 for various models as determined from the $F 450 W$ WFPC 2 image. See text for details. The best fitting model (lowest $\chi^{2}$ ) is shown in bold.

\begin{tabular}{ccccc}
\hline \hline WFPC2 & Type & Index & $R_{\text {eff }}(\mathrm{pc})$ & $\chi^{2} / \chi_{\text {best }}^{2}{ }^{b}$ \\
\hline & Gauss & - & 4.34 & 1.93 \\
& King & 5 & 4.27 & 1.54 \\
& King & 15 & 3.89 & 1.13 \\
& King & 30 & 3.66 & 1.24 \\
& King & 100 & 4.14 & 1.87 \\
& Moffat & 1.5 & 4.34 & 1.09 \\
& Moffat & 2.5 & 4.09 & 1.14 \\
\hline ACS & Moffat $^{a}$ & $\mathbf{1 . 7 6}$ & $\mathbf{4 . 0 8}$ & $\mathbf{1 . 0}$ \\
\hline & Filter & Type & index & $R_{\text {eff }}$ \\
\hline & $F 555 W$ & Moffat & 1.76 & $4.83 \pm 0.25$ \\
& $F 814 W$ & Moffat & 1.76 & $3.68 \pm 0.1$ \\
\hline
\end{tabular}

a Best fitting model of the WFPC2 observations.

${ }^{b}$ Calculated individually for the different cameras.

Table 2. Effective radius of NGC 7252:W30 for various models as determined from the $F 555 \mathrm{~W}$ WFPC2 image (PC chip). See text for details. The best fitting model (lowest $\chi^{2}$ ) is shown in bold.

\begin{tabular}{ccccc}
\hline \hline WFPC2 & Type & Index & $R_{\text {eff }}(\mathrm{pc})$ & $\chi^{2} / \chi_{\text {best }}^{2}$ \\
\hline Gauss & - & 13.2 & 3.67 \\
King & 5 & 12.5 & 2.93 \\
King & 15 & 11.0 & 1.82 \\
King & 30 & 10.2 & 1.31 \\
King & $\mathbf{1 0 0}$ & $\mathbf{8 . 3}$ & $\mathbf{1 . 0 0}$ \\
Moffat & 1.5 & 11.0 & 1.21 \\
Moffat & 2.5 & 12.0 & 2.19 \\
\hline
\end{tabular}

in a wavelength coverage from $4200 \AA$ to $6200 \AA$ and a resolution of $\approx 5 \mathrm{~km} \mathrm{~s}^{-1}$ at $5200 \AA$. The data were reduced and extracted using the on-line UVES pipeline with the relevant bias and flat-frames. Cosmic-rays were also removed using the pipeline. After extraction, each spectrum was corrected to the helio-centric velocity frame, and summed to create the total spectrum for each cluster. The total exposure times were $8.67 \mathrm{~h}$ on G114 and $25.1 \mathrm{~h}$ on W30. Figures 1 and 2 show the positions and lengths of the UVES slits for clusters W30 and G114 respectively, superimposed on HST planetary camera images. We note that the background near the positions of each of these clusters is devoid of spurious sources, which allowed a clear background subtraction (determined using a spline function) at the position of the clusters.

Additionally, we observed several template stars which were used to complement our existing template catalogue (Maraston et al. 2004), namely HR 35 (F4 V), HR 8709 (A4 V), HD 203638 (K0 III), and HD 212574 (A6 V) where the designation in the brackets refers to the spectral type of the star. The stars were reduced in the same way as described above. 
Table 3. Velocity dispersion measurements of NGC 1316:G114.

\begin{tabular}{ccccccc}
\hline \hline Template & Spectral type & \multicolumn{5}{c}{$\sigma\left(\mathrm{km} \mathrm{s}^{-1}\right)$} \\
& & $4360-5115 \AA$ & $5245-5432 \AA$ & $5486-5855 \AA$ & $5943-6113 \AA$ & Average \\
\hline HR 35 & F4 V & 41.8 & 47.3 & 43.9 & 43.4 & 44.1 \\
HD 203638 & K0 III & 43.6 & 41.9 & 48.0 & 47.6 & 45.3 \\
${\text { Template } 1^{a}}^{c}$ & - & 39.9 & 36.3 & 41.0 & 45.6 & 40.7 \\
Template $^{b}$ & - & 40.3 & 37.8 & 40.7 & 44.7 & 40.9 \\
Template 3 $^{c}$ & - & 41.1 & 41.4 & 45.2 & 45.6 & 43.4 \\
\hline
\end{tabular}

a $50 \% \mathrm{~K} 0 \mathrm{III}$ star and $50 \% \mathrm{~F} 4 \mathrm{~V}$ star.

${ }^{b} 60 \% \mathrm{~K} 0 \mathrm{III}$ star and $40 \% \mathrm{~F} 4 \mathrm{~V}$ star.

c $70 \% \mathrm{~K} 0 \mathrm{III}$ star and $30 \% \mathrm{~F} 4 \mathrm{~V}$ star.

The determination of the velocity dispersion of each of the clusters was carried out in exactly the same way as was done for W3, which is described in detail in Maraston et al. (2004). In summary we used an adapted version of the Fourier Correlation Quotient (FCQ, Bender 1990) method as implemented by Bender et al. (1994), using templates chosen to match the stellar populations within each cluster. The templates were chosen to have temperatures and gravities (i.e. luminosity classes) appropriate to stars at the main-sequence turn-off point and giants stars in synthetic stellar populations of the same age as each cluster. Their contributions to the composite template were weighted using the weights predicted by the SSPs at the appropriate age (Maraston 2005, e.g. her Fig. 13).

\subsection{NGC 1316:G114}

The results of the determination of the velocity dispersion for G114 is shown in Table 3. Due to the high $\mathrm{S} / \mathrm{N}$ ratio of the data and the large number of metal lines in the optical part of the spectrum (due to the dominance of cool stars at the cluster's age of $\sim 3 \mathrm{Gyr}$ ) we were able to use the full spectral range to determine the velocity dispersion. We note that the results do not depend crucially on the assumed stellar template. The adopted one-dimensional velocity dispersion for G114 is $\sigma(G 114)=42.1 \pm 2.8 \mathrm{~km} \mathrm{~s}^{-1}$, which is the average over the full wavelength range of Templates 2 and 3 , which should be the closest match to the actual stellar population based on the models of Maraston (2005). Figure 3 shows the blue section of the observed spectrum of G114 (black), the best fitting broadened stellar template (red) and the difference between the two (green). All spectra shown in this work have been divided by the continuum and had a value of one subtracted from them, to place the average value at zero.

We have also measured the heliocentric line-of-sight velocity of G114,

$v(G 114)=1292 \pm 3 \mathrm{~km} \mathrm{~s}^{-1}$.

This is consistent with the results of Goudfrooij et al. (2001a), who measured $1306 \pm 26 \mathrm{~km} \mathrm{~s}^{-1}$.

\subsection{NGC 7252:W30}

Based on comparisons between the optical/near-infrared colours (Miller et al. 1997; Maraston et al. 2001) and optical spectroscopy (Schweizer \& Seitzer 1998), W30 and W3 appear to have very similar ages and metallicities. Because of this, we have used the same stellar template to determine the velocity dispersion of W30 as we used for W3 (Maraston et al. 2004). Similarly as was done for W3, we have limited our analysis to the region red-ward of $\mathrm{H} \beta$, in particular concentrating on the region around the $\mathrm{Mg}$ triplet $(\lambda \lambda 5167,5172,5183 \AA)$ and Fe lines $(\lambda \lambda 5270,5335 \AA$ ). Table 4 shows the results of the fitting on specific regions of the spectrum. We adopt the value $\sigma(W 30)=27.5 \pm 2.5 \mathrm{~km} \mathrm{~s}^{-1}$ which is the average between the value found for fitting solely on the $\mathrm{Mg}$ lines and fitting on the full region of interest (between $5150 \AA$ and $5350 \AA$ ).

Figure 4 shows the spectrum of cluster W30 in the fitting region (black), the best fitting broadened template (red), and the difference between the two (green).

The measured line of sight velocity of W30 is $v(W 30)=4614 \pm 1 \mathrm{~km} \mathrm{~s}^{-1}$,

which is in very good agreement with previous measurements, namely $4624 \pm 17 \mathrm{~km} \mathrm{~s}^{-1}$ (Schweizer \& Seitzer 1998).

\section{Dynamical masses}

Assuming that the clusters are in virial equilibrium, we can estimate their virial masses using the relation

$\mathcal{M}_{\mathrm{dyn}}=\eta \frac{\sigma_{\mathrm{x}}^{2} r_{\mathrm{eff}}}{G}$

(Spitzer 1987, p. 11-12) where $\eta$ is a dimensionless parameter which depends on the cluster profile adopted. Here we adopt $\eta=9.75$, which is approximately valid for most of the globular clusters in the Milky Way. However, we will return to this adopted value, and its consequences in Sect. 5.1.1. Inserting the numbers derived above we find that

$$
\begin{aligned}
& \mathcal{M}_{\text {dyn }}(G 114)=1.64( \pm 0.13) \times 10^{7} \mathcal{M}_{\odot} \\
& \mathcal{M}_{\text {dyn }}(W 30)=1.59( \pm 0.26) \times 10^{7} \mathcal{M}_{\odot}
\end{aligned}
$$



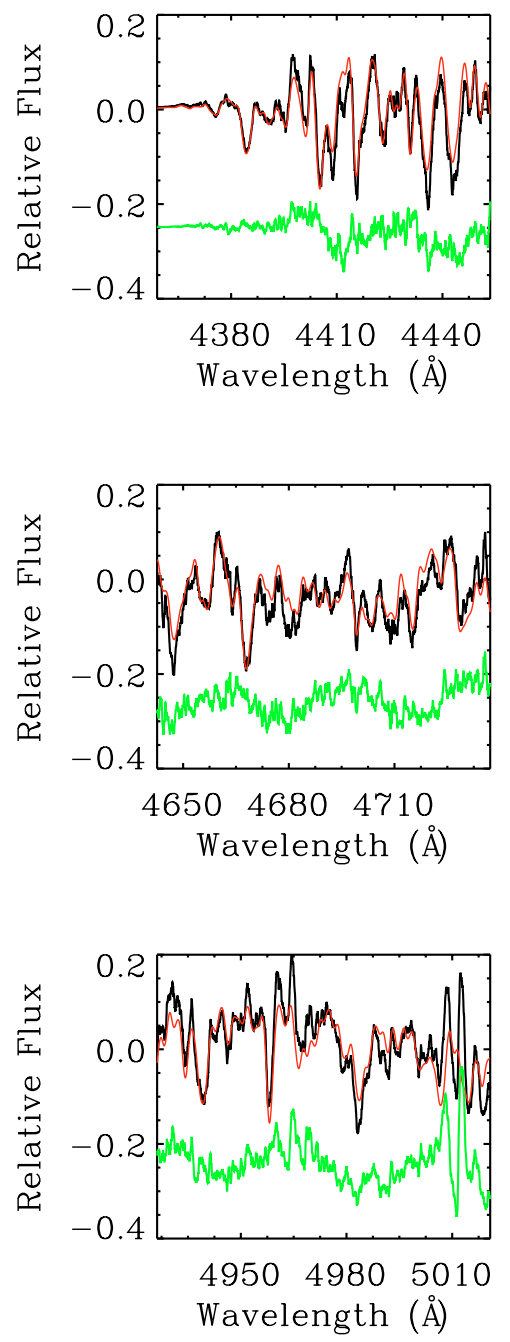
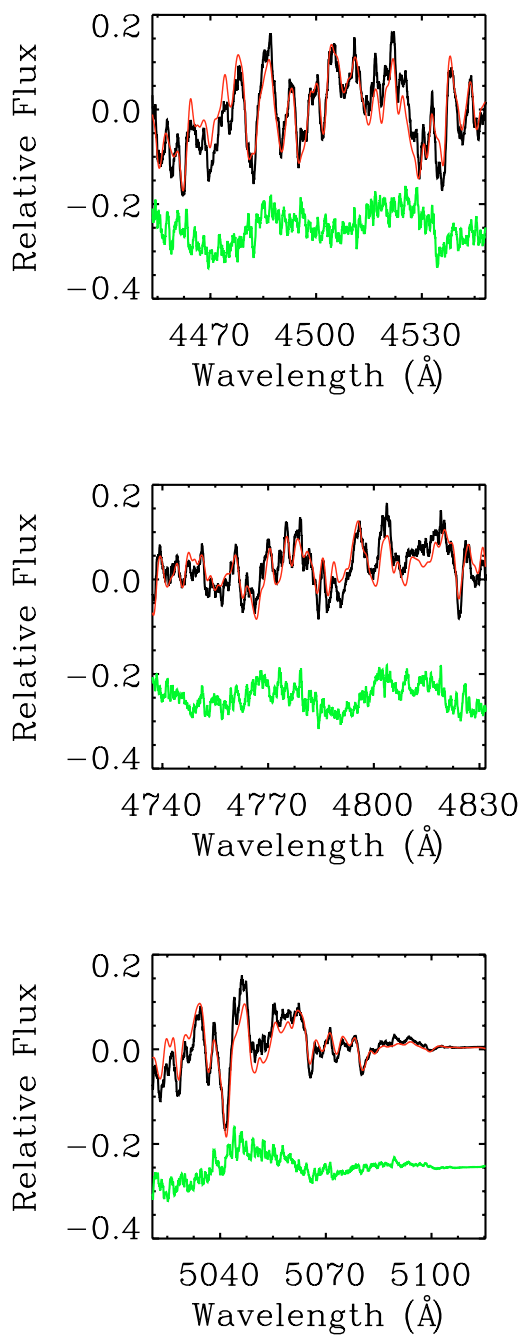
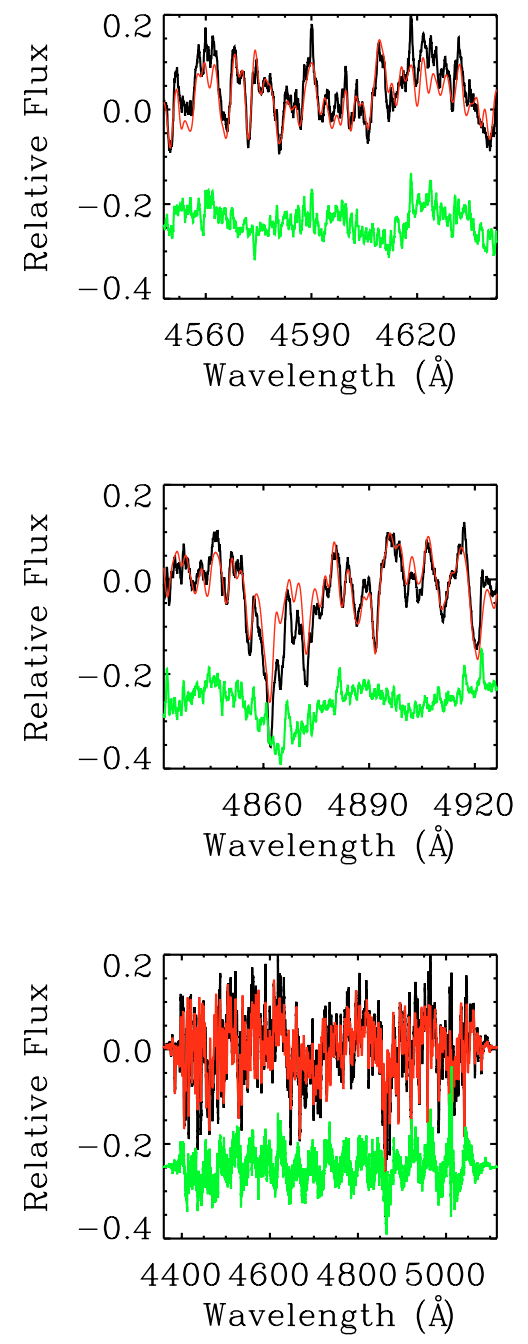

Fig. 3. The observed (observed divided by the continuum minus 1 and smoothed by 15 pixels) spectrum of G114 is plotted in black. The red shows the best fitting template, broadened by $42.1 \mathrm{~km} \mathrm{~s}^{-1}$. The green is the difference between the two, shifted downward by a constant for clarity. This is just one region that we fitted, Table 3 shows all the regions that were used. The large scale undulations $(\sim 30 \AA)$ are due to the response of the individual orders of the echelle spectra. Since the scale length of these variations is much larger than those of the spectral features of interest $(<5 \AA)$ they do not affect the derived velocity dispersion.

Table 4. Derived velocity dispersion for different portions of the spectra of W30 in NGC 7252. All values are given in $\mathrm{km} \mathrm{s}^{-1}$.

\begin{tabular}{cccc}
\hline \hline $\mathrm{Mg}^{a}$ & $\mathrm{Fe}^{b}$ & Full $^{c}$ & Adopted \\
\hline 24.7 & 30.3 & 28.0 & $27.5 \pm 2.5$ \\
\hline
\end{tabular}

a Just the Mg triplet, from $5156 \AA$ to $5187 \AA$.

$b$ Red-ward of the $\mathrm{Mg}$ triplet, mainly dominated by Fe lines, from $5218 \AA$ to $5351 \AA$.

c The full spectrum used, from $5155 \AA$ to $5324 \AA$.

where the errors do not include uncertainties in the distance to the clusters. The adopted properties of W3, W30, and G114 are shown in Table 5.

We note, however, that the value of the constant in Eq. (1) known as $\eta$, may change as a function of age of the cluster (Boily et al. 2005). This effect will be most dramatic in the first 30 Myr of a cluster's lifetime, and the size of the effect depends on the surface mass density of the cluster in the sense that the clusters with the highest densities will be the most affected. We will return to this point in Sect. 5.1.1.

These results confirm the extremely large masses of G114 and W30. However, it is important to put these clusters in the context of the mass functions of the full cluster systems of their respective galaxies in order to test to what extent they really are outliers $^{1}$.

To do this we have performed a series of monte-carlo tests of the mass function of cluster populations. We assume an initial power-law mass distribution of the clusters within each galaxy of the form $N(\mathcal{M}) \mathrm{d} \mathcal{M} \propto \mathcal{M}^{-\alpha} \mathrm{d} \mathcal{M}$, with $\alpha \sim 2$ (e.g. Miller et al. 1997) and also that this distribution gets filled randomly. In the case of the NGC 7252 system, W3 (the most

1 As noted in Miller et al. (1997) and Goudfrooij et al. (2001a,b) both NGC 7252 and NGC 1316 have large populations of luminous star clusters which appear to be coeval with the merger of the host galaxies. 

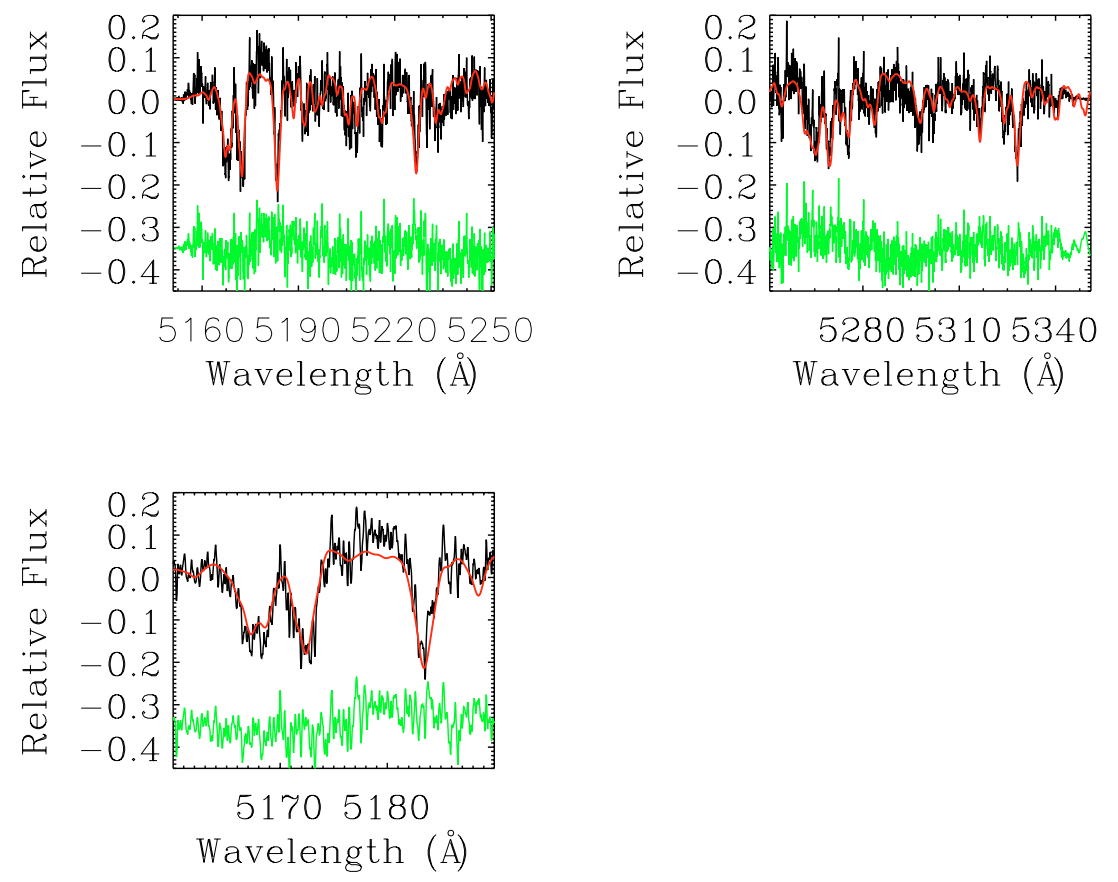

Fig. 4. The observed (smoothed by 5 pixels) spectrum of W30 is plotted in black. The red shows the best fitting template, broadened by $28.0 \mathrm{~km} \mathrm{~s}^{-1}$. The green in the difference between the two, shifted downward by a constant for clarity. The top two panels show the entire wavelength range which was used in the fitting, while the bottom figure shows a blow up of the region including the Mg triplet, which are the strongest lines in this region.

Table 5. The properties of the massive star clusters.

\begin{tabular}{cccccc}
\hline \hline Name & $\begin{array}{c}M_{V}{ }^{a} \\
(\mathrm{mag})\end{array}$ & $\begin{array}{c}R_{\text {eff }} \\
(\mathrm{pc})\end{array}$ & $\begin{array}{c}\sigma \\
\left(\mathrm{km} \mathrm{s}^{-1}\right)\end{array}$ & $\begin{array}{c}\text { age } \\
\mathrm{Gyr}\end{array}$ & $\begin{array}{c}\text { Mass } \\
\left(10^{7} \mathcal{M}_{\odot}\right)\end{array}$ \\
\hline NGC 7252:W3 & -16.2 & $17.5 \pm 1.8$ & $45 \pm 5$ & $0.4 \pm 0.15$ & $8 \pm 2$ \\
NGC 7252:W30 & -14.6 & $9.3 \pm 1.7$ & $27.5 \pm 2.5$ & $0.4 \pm 0.15$ & $1.59 \pm 0.26$ \\
NGC 1316:G114 & -13.0 & $4.08 \pm 0.55$ & $42.1 \pm 2.8$ & $3 \pm 1$ & $1.64 \pm 0.13$ \\
\hline
\end{tabular}

$a$ We have corrected for foreground extinction of NGC 7252 and NGC 1316 of $A_{V}=0.03$ and $A_{V}=0.0$ respectively.

massive cluster in the NGC 7252) is $\sim 5$ times more massive than W30 (the second most massive cluster in this system). Under these conditions, we expect that $\sim 20 \%$ of the realizations of the cluster populations will have a factor of five or greater between the most massive and the second most massive clusters within the system ${ }^{2}$. The third brightest cluster in NGC 7252 system (W6) is $\sim 0.2$ mag fainter than W30, corresponding to a mass difference of only $\sim 17 \%$ (assuming a common age). Hence, we see that while W3 and W30 are extremely massive clusters, they are compatible with being the most massive clusters of a continuous power-law distribution, which we note continues to the detection limit.

The intermediate aged cluster population of NGC 1316 can also be readily explained by the same argument as above, as the second and third most massive clusters in this system (G114 is the most massive) are only 0.47 and 0.54 mag fainter respectively. This corresponds to less than a factor of two in the luminosity (and mass assuming that the clusters have similar ages

\footnotetext{
2 We have also implicitly assumed that the ratio between the lowest mass cluster and the highest mass cluster is $\ll 0.001$.
}

and metallicities). A difference of two or greater in the ratio of the most massive and second most massive clusters was found in $\sim 50 \%$ of the realizations of a cluster population. Goudfrooij et al. (2004) have reported that the bright end of the luminosity function (which we assume to represent the mass function) is well approximated by a power-law of the type used above.

Since NGC 7252:W3, NGC 7252:W30, and NGC 1316:G114 can be readily understood through sampling statistics we will assume that they are simply the most massive clusters of a continuous distribution. In Sect. 5.2 we will use this assumption and the detailed properties of these clusters to understand more enigmatic objects, namely the dwarf galaxy transition objects (DGTOs) and ultra-compact dwarf galaxies (UCDs).

\section{Discussion}

\subsection{Stellar mass functions}

A comparison between the mass of a cluster derived using photometric methods and the mass determined through kinematic 
arguments, allows an independent test of the assumptions that went into each estimate. The assumption that has garnered the most attention in recent years, is that of the underlying stellar mass function (MF) of the cluster. In order to estimate the photometric mass of a cluster, mass-to-light ratios from simple stellar population (SSP) models are required. These, in turn, are heavily dependent on the assumed stellar mass function. Therefore, assuming that all the other assumptions are valid (such as the state of equilibrium, correct extinction determination, and stellar evolutionary tracks) any difference between the mass of a cluster derived in these two ways is caused by the difference between the input stellar mass function and that of the cluster.

Studies that have used this technique have reported strongly divergent results. For example, Smith \& Gallagher (2001) and McCrady et al. (2005) have reported that the YMC M82F is deficient in low-mass stars, relative to the standard Salpeter (1955) (a single power-law mass distribution from the lower to the upper mass limit) or Kroupa (2002) type MFs (a single power-law above $1 \mathcal{M}_{\odot}$ and significantly flatter below this limit). However, Maraston et al. (2004), Larsen et al. (2004), and Larsen \& Richtler (2004) have shown that clusters in a wide variety of galactic environments are consistent with a Salpeter or Kroupa-type MF.

We therefore carry out this experiment for the two massive clusters W30 and G114. In Fig. 5 we show the light-to-mass ratio from the Maraston (2005) models for solar metallicity and a Salpeter (dashed line) \& Kroupa (solid line) stellar mass functions. Over-plotted in red are the two clusters in the present study as well as W3 from our previous study. These three clusters all lie impressively close to the value using a Kroupa MF. Hence they are most likely not deficient in low mass stars.

\subsubsection{Cluster measurements from the literature}

In order to compare our results to other young clusters, we have taken a sample of clusters with velocity dispersion and radii measurements from the literature. The clusters, their parameters, and the corresponding references are listed in Table 6. We have taken the fundamental parameters (age, extinction, brightness, velocity dispersion, radius, and distance modulus) directly from the given reference. In some cases the $V$-band magnitude was not given, although we note that all clusters with ages greater than $20 \mathrm{Myr}$ have observed $V$-band magnitudes. These older clusters will constitute the main part of our comparison. In those cases where $V$-band magnitudes were not available we transformed the given magnitude to the $V$-band using the colours in the Maraston (2005) SSP models at the appropriate age (which assume a Salpeter IMF).

We have estimated the mass of each cluster using Eq. (1) and used their $V$-band magnitudes (and given ages) to place them in Fig. 5 (blue points). From this figure it is clear that the amount of deviation from standard stellar mass functions (Kroupa or Salpeter-type) is heavily age dependent, with the

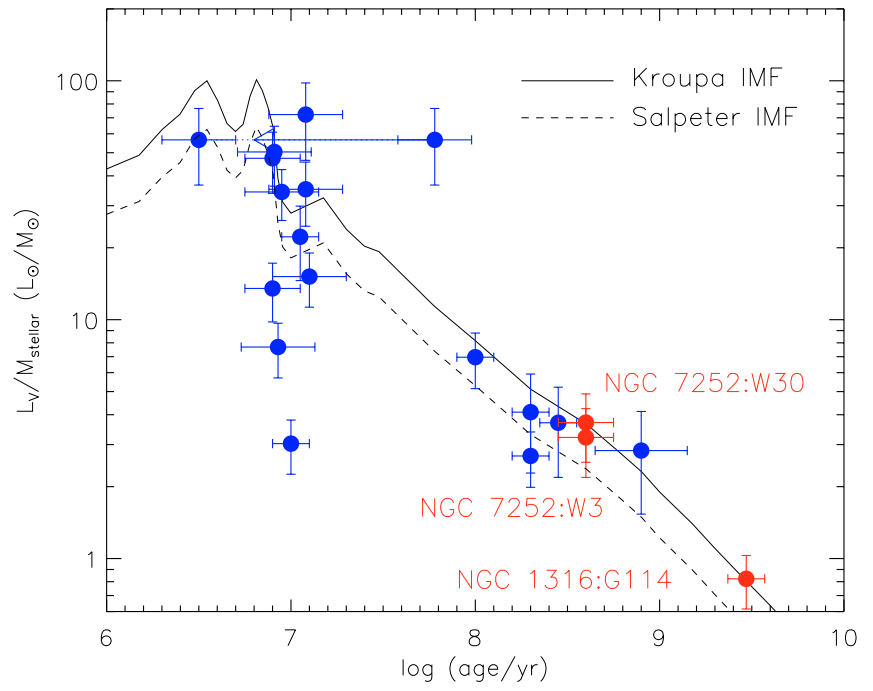

Fig. 5. The derived light-to-mass ( $V$-band) ratios as a function of the age of the clusters. Over-plotted are the $L / \mathcal{M}$ ratios (for solar metallicity) from the Maraston (2005) SSP models with a Kroupa (solid line) and Salpeter (dashed line) stellar mass function. The clusters older than a few $\times 10^{7}$ Myr (except M82F) are well fit by the models, whereas some the youngest clusters show significant deviation. This may be caused by a strongly varying $\eta$ in the early stages of a cluster's lifetime (Boily et al. 2005). For M82F, we show the position of this cluster for two ages, $4 \mathrm{Myr}$ and $60 \mathrm{Myr}$ connected with a dotted line.

older clusters (with the exception of $\mathrm{M} 82 \mathrm{~F}^{3}$ ) all consistent with a Kroupa or Salpeter-IMF and the youngest clusters showing a large amount of scatter. Below we outline three possible explanations for this.

A first possibility for the age-dependent scatter in Fig. 5 is that $\eta$ (the parameter in the numerator of Eq. (1)) changes as a function of time (Boily et al. 2005). This is caused by mass-segregation in young clusters and further internal dynamical evolution of the cluster. The variation of $\eta$ is expected to also be heavily dependent on the surface density of the star cluster, with higher surface densities leading to larger variations of $\eta$. Figure 6 shows the mean surface density within the half-light radius (using the estimated virial mass of the cluster) of star clusters vs. their measured velocity dispersions. The small blue filled triangles represent old globular clusters in our galaxy (McLaughlin \& van der Marel 2005), the small green filled circles are globular clusters in M 31 (a collation of data from McLaughlin \& van der Marel in prep.), the small upsidedown magenta triangles are old GCs in NGC 5128 (Martini $\&$ Ho 2004), and the large red circles are young massive star clusters in a variety of galaxies (listed in Table 6). All of the YMCs in Fig. 6 have surface densities above $10^{3} \mathcal{M}_{\odot} \mathrm{pc}^{-2}$ and most are above $10^{4} \mathcal{M}_{\odot} \mathrm{pc}^{-2}$, which is the regime where $\eta$ is expected to vary strongly (Boily et al. 2005).

\footnotetext{
${ }^{3}$ M82-F, located in Fig. 5 at log (age) $=7.8$, may be a deviant point in the diagram due to uncertainties in its age and extinction. Further studies to pin down the exact values would be desirable. Additionally, we note that its elliptical shape, crowded environment, and peculiar radial velocity suggest that the cluster may have been gravitationally influenced by its surroundings, and hence may not be in virial equilibrium.
} 
Table 6. The properties of young massive clusters taken from the literature.

\begin{tabular}{|c|c|c|c|c|c|c|c|c|c|}
\hline Galaxy & ID & $\begin{array}{c}\text { Age } \\
(\log y r)\end{array}$ & $\begin{array}{c}\Delta \text { age } \\
(\log y r)\end{array}$ & $\begin{array}{c}M_{\mathrm{V}} \\
(\mathrm{mag})\end{array}$ & $\begin{array}{l}R_{\text {eff }} \\
(\mathrm{pc})\end{array}$ & $\begin{array}{l}\Delta R_{\text {eff }} \\
(\mathrm{pc})\end{array}$ & $\begin{array}{c}\sigma \\
\left(\mathrm{km} \mathrm{s}^{-1}\right)\end{array}$ & $\begin{array}{c}\Delta \sigma \\
\left(\mathrm{km} \mathrm{s}^{-1}\right)\end{array}$ & Reference $^{a}$ \\
\hline \multicolumn{10}{|c|}{ Young Massive Star Clusters } \\
\hline M 82 & $\mathrm{~F}$ & $7.6(6.6)$ & 0.17 & -14.2 & 1.5 & 0.5 & 13.4 & 0.7 & 1,10 \\
\hline M 82 & MGG9 & 6.9 & 0.15 & -13.43 & 2.6 & 0.4 & 15.9 & 0.8 & 4 \\
\hline M 82 & MGG11 & 6.9 & 0.15 & -13.23 & 1.2 & 0.17 & 11.4 & 0.8 & 4 \\
\hline NGC 1569 & A & 7.08 & 0.2 & -14.1 & 1.9 & 0.2 & 15.7 & 1.5 & 7,8 \\
\hline NGC 1705 & 1 & 7.08 & 0.2 & -14.0 & 1.6 & 0.2 & 11.4 & 1.5 & 1,9 \\
\hline NGC 4038 & W99-15 & 6.93 & 0.2 & -13.69 & 3.6 & 0.5 & 20.2 & 0.7 & 6 \\
\hline NGC 4038 & W99-2 & 6.95 & 0.2 & -14.79 & 4.5 & 0.5 & 14.2 & 0.4 & 6 \\
\hline NGC 4038 & W99-1 & 6.91 & 0.2 & -14.0 & 3.6 & 0.5 & 9.1 & 0.6 & 6 \\
\hline NGC 4038 & W99-16 & 7.0 & 0.1 & -12.70 & 6.0 & 0.5 & 15.8 & 1.0 & 6 \\
\hline NGC 4214 & 10 & 8.3 & 0.1 & -10.22 & 4.33 & 0.1 & 5.1 & 1.0 & 3 \\
\hline NGC 4214 & 13 & 8.3 & 0.1 & -11.68 & 3.01 & 0.2 & 14.8 & 1.0 & 3 \\
\hline NGC 4449 & 27 & 8.9 & 0.25 & -9.61 & 3.72 & 0.32 & 5.0 & 1.0 & 3 \\
\hline NGC 4449 & 47 & 8.45 & 0.10 & -10.74 & 5.24 & 0.76 & 6.2 & 1.0 & 3 \\
\hline NGC 5236 & 502 & 8.0 & 0.1 & -11.57 & 7.6 & 1.1 & 5.5 & 0.2 & 2 \\
\hline NGC 5236 & 805 & 7.1 & 0.2 & -12.17 & 2.8 & 0.4 & 8.1 & 0.2 & 2 \\
\hline NGC 6946 & 1447 & 7.05 & 0.10 & -14.17 & 10.2 & 1.6 & 8.8 & 1.0 & 3 \\
\hline NGC 1316 & G114 & 9.47 & 0.1 & -13.0 & 4.1 & 0.25 & 42.1 & 2.8 & this work \\
\hline NGC 7252 & W30 & 8.6 & 0.1 & -14.6 & 9.3 & 1.7 & 27.5 & 2.5 & this work \\
\hline NGC 7252 & W3 & 8.6 & 0.1 & -16.2 & 17.5 & 1.8 & 45 & 5.0 & 5 \\
\hline Nuclear Star Clusters & & & & $M_{I}^{b}$ & & & & & \\
\hline NGC 300 & - & - & - & -11.4 & 2.9 & - & 13.3 & 2.0 & 11,12 \\
\hline NGC 428 & - & - & - & -13.1 & 3.4 & - & 24.4 & 3.7 & 11,12 \\
\hline NGC 1042 & - & - & - & -13.1 & 1.9 & - & 32.0 & 4.8 & 11,12 \\
\hline NGC 1493 & - & - & - & -13.1 & 2.6 & - & 25.0 & 3.8 & 11,12 \\
\hline NGC 3423 & - & - & - & -11.8 & 4.2 & - & 30.4 & 4.6 & 11,12 \\
\hline NGC 7793 & - & - & - & -13.6 & 7.7 & - & 24.6 & 3.7 & 11,12 \\
\hline
\end{tabular}

a References: 1) Smith \& Gallagher (2001); 2) Larsen \& Richtler (2004); 3) Larsen et al. (2004); 4) McCrady et al. (2003); 5) Maraston et al. (2004); 6) Mengel et al. (2002); 7) Ho \& Filippenko (1996b); 8) Anders et al. (2004); 9) Ho \& Filippenko (1996a); 10) McCrady et al. (2005); 11) Böker et al. (2004); 12) Walcher et al. (2005).

${ }^{b}$ For Fig. 7 we have assumed $(B-I)=1.0$ which corresponds to a simple stellar population of $\sim 400 \mathrm{Myr}$ and solar metallicity, although we note that the assumed $(B-I)$ colour does not change the conclusions.

A second possibility is that many of the youngest clusters are not in dynamical equilibrium. This could be due to external gravitational effects (e.g. close passages to massive GMCs). As clusters are born in gas-rich environments this is a likely possibility. A lack of equilibrium could also be caused by the rapid expulsion of the gas left over from the star formation process (assuming a non-100\% star formation efficiency). This can have a severe influence on a young cluster (e.g. Boily \& Kroupa 2003). Bastian et al. (2005a) have suggested that rapid gas removal may be responsible for the dissolution of $70-90 \%$ of clusters within the first $10 \mathrm{Myr}$ of their lives, independent of cluster mass. The lack of dynamical equilibrium is also supported by the work of de Grijs et al. (2005) who showed that the clusters which deviate the most from the old globular cluster $M_{V}$ vs. $\log (\sigma)$ relation are found in the highest density environments, and hence are the most likely to be affected by interactions with the external environment.

Finally, a third possibility for the observed age-dependent scatter in Fig. 5 is that only clusters with Kroupa or Salpetertype stellar mass functions survive for more than $\sim 100 \mathrm{Myr}$. Smith \& Gallagher (2001) suggest that if the cluster M82 F has a significantly top-heavy stellar IMF (i.e. truncated below 2-3 $M_{\odot}$ ) it will lack the gravitational potential to remain bound due to stellar evolutionary mass loss after 2-3 Gyr. However, in Fig. 5 the clusters which deviate the most from Salpter or Kroupa-type stellar IMFs have light-to-mass ratios below the expected value. This implies that they are overabundant in low mass stars relative to Salpeter or Kroupa-type IMFs. Since low mass, long-lived stars provide the gravitational potential to keep a cluster bound, we would expect these 


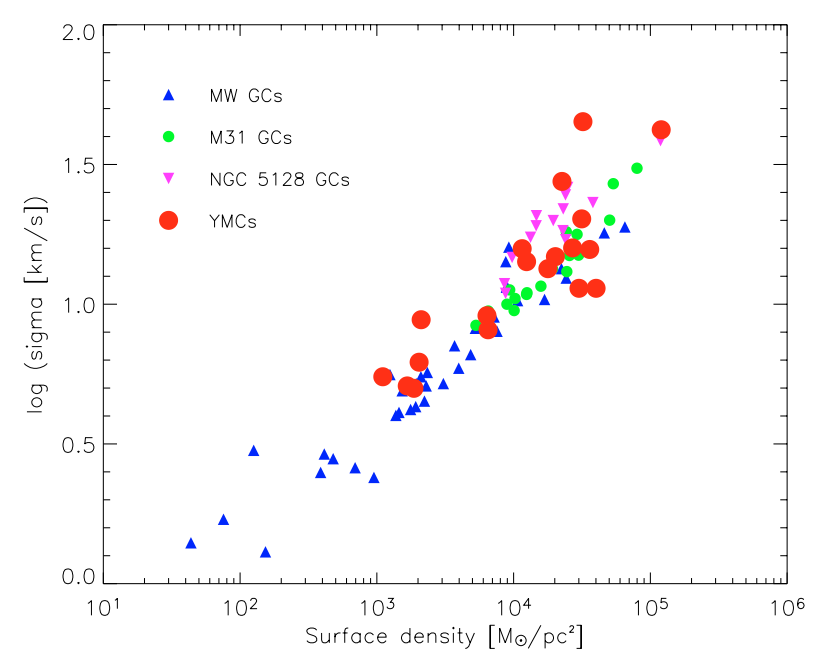

Fig. 6. The measured velocity dispersion of star clusters versus their mean surface density within the half light radius. The blue triangles, small green circles, magenta upside-down triangles and the large red circles represent old globular clusters in the Galaxy, M 31, NGC 5128, and young massive star clusters in a variety of galaxies, respectively. Note that the masses have been estimated assuming virial equilibrium. However, many of the young clusters have surface densities greater than $10^{4} \mathcal{M}_{\odot} / \mathrm{pc}^{2}$, which is the regime where $\eta$ is expected to vary significantly (Boily et al. 2005).

clusters to be long-lived and hence to see old clusters which also have light-to-mass ratios below that expected for standard IMFs. Such clusters are clearly lacking in Fig. 5 arguing against this possibility.

\subsection{More clues from $\kappa$-space}

\subsubsection{The future evolution of $\mathrm{W} 30$ and $\mathrm{G} 114$}

Following the analysis by Maraston et al. (2004) we attempt to place W30 and G114 in the broader context of gravitationally bound stellar systems. For this we exploit the re-definition of the fundamental plane known as $\kappa$-space (Bender et al. 1992) which combines the three fundamental observable parameters (radius, surface brightness, and velocity dispersion) into physically motivated values.

Figure 7 shows the position of W3 (asterisk), W30 (upward triangle), and G114 (downward triangle) in the $\kappa 1-\kappa 2$ plane. In this space $\kappa 1$ traces the mass of the system, while $\kappa 2$ measures the compactness of a system for a given mass (the product of $\mathcal{M} / \mathrm{L}$ and surface brightness). For comparison we also show the mean positions of bulges and ellipticals $(B+E)$, dwarf ellipticals (dE), M32 (all taken from Burstein et al. 1997, and assuming $H_{0}=75 \mathrm{~km} \mathrm{~s}^{-1} \mathrm{Mpc}^{-1}$ ). Next, we add the point-like objects in Fornax (the ultra-compact dwarf galaxies or UCDs) (the average value of the four objects presented in Drinkwater et al. 2003). We also plot the positions of old globular clusters in the Milky Way (small blue triangles) (McLaughlin \& van der Marel 2005), M 31 (small green dots) (a collation of data from McLaughlin \& van der Marel in prep.), and NGC 5128 (magenta up-side triangles) (Martini \& Ho 2004), for which we assumed a constant $(B-V)$ colour for old metal

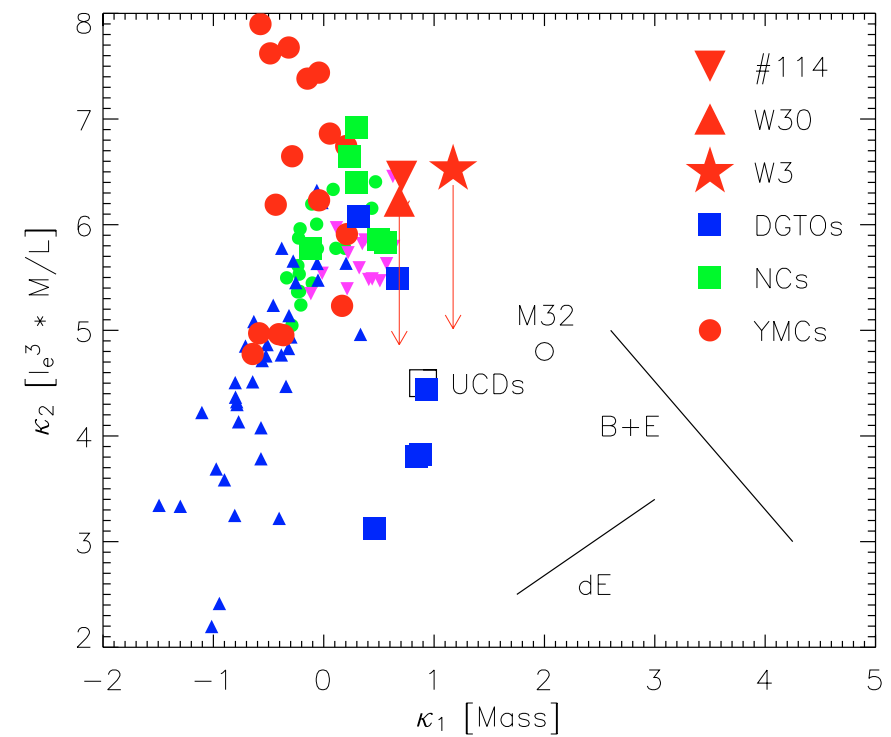

Fig. 7. $\kappa$-space: the $\kappa$ space of stellar systems, in a remake of Fig. 3 of Maraston et al. (2004, see text for the definitions of $\kappa 1$ and $\kappa 2$ ). W30 and G114 are shown as red triangles. The arrows show how the clusters will move in this space after ageing them to a common age of $10 \mathrm{Gyr}$ (using SSP models). Large filled red circles are young clusters taken from the literature (see Table 6). Small filled blue triangles, green cicles, and magenta upside-down triangles are GCs from the Galaxy, M31, and NGC 5128 respectively (see text). The filled green squares represent the position of nuclear star clusters and the filled blue squares are the dwarf galaxy transition objects (DGTOs) found in the Virgo cluster (note that we assume a $(B-V)=1.0$ for these objects, typical of a solar metallicity $10 \mathrm{Gyr}$ old stellar population) found in the Virgo cluster. The positions of other self-gravitating objects are shown, ultra-compact dwarf galaxies (UCDs), M32, Bulges and Ellipticals (B+E), and dwarf Ellipticals (dE).

poor GCs, namely $0.7 \mathrm{mag}$. We also add the young clusters taken from the literature (see Table 6), which are shown as red points. The large filled green squares represent the Nuclear Star Clusters (NCs) in bulge-less disk galaxies (Böker et al. 2004; Walcher et al. 2005, see Table 6 for details). Finally, we add the dwarf galaxy transition objects (DGTOs) found in the Virgo galaxy cluster (Haşegan et al. 2005) as filled blue squares. For the DGTOs we assumed that $(B-V)=1$, typical of a $10 \mathrm{Gyr}$ solar metallicity SSP.

The arrows which begin at W3, W30, and G114 represent the evolution of the cluster in this space when the clusters are "aged" to a common age of $10 \mathrm{Gyr}$ using the Maraston (2005) SSP models. Note, however, that the SSP model tracks do not take mass loss (and hence fading) by evaporation or due to external perturbations into account.

As was found for the massive cluster NGC 7252:W3 by Maraston et al. (2004), we find that NGC 7252:W30 and NGC 1316:G114 will evolve into the region of $\kappa$-space occupied by the UCDs and DGTOs. This shows a strong similarity between the most massive star clusters and these enigmatic objects, and may suggest that they formed through similar mechanisms.

Additionally, we can estimate the amount of mass loss which is expected to occur within W30 and G114. From the 
SSP models of Maraston (2005) we see that a cluster (assuming solar metallicity and a Kroupa stellar IMF) is expected to lose $\sim 18 \%$ of its mass between the ages of $400 \mathrm{Myr}$ and $10 \mathrm{Gyr}$ (i.e. between the present age of W30 and the age of globular clusters). G114, with an age of $\sim 3$ Gyr is only expected to lose $\sim 5 \%$ of its current mass to stellar evolution. Using the analytic expressions for mass loss in a tidal field of Lamers et al. (2005, Eq. (6)), we note that due to the strong dependence on cluster mass, neither of the clusters studied here are expected to lose a significant amount of mass due to disruption. W30 is expected to lose $\sim 8 \%$ of its mass over the next $10 \mathrm{Gyr}$ while G114 is expected to lose just $\sim 5 \%$ of its mass over the next 7 Gyr. For this calculation we have assumed $t_{4}$ (the average time for a $10^{4} M_{\odot}$ cluster to disrupt) to be $1 \mathrm{Gyr}$, based on the galactic GC population (Boutloukos \& Lamers 2003). However, we note that the conclusions reached are not significantly affected by the choice of $t_{4}$. As these small changes would barely be visible in Fig. 7 and would only add confusion, we choose not to show them.

\subsubsection{The relation between YMCs and old globular clusters}

Figure 8 again shows the $\kappa 1-\kappa 2$ projection of $\kappa$-space, except with all of the YMCs (red points) aged to $10 \mathrm{Gyr}$ using SSP models, again assuming only passive stellar evolution of the cluster. Here we see that many of the YMCs have evolved "past" the globular cluster region and into the space occupied by W3, W30, the UCDs and the UGTOs. Burstein et al. (1997) suggest that the tightness of the GC relation in $\kappa$-space may be due to the preferential destruction of star clusters outside a rather narrow region of parameter space (e.g. mass and radius, see also Fall \& Rees 1977). Along the same lines, Gnedin \& Ostriker (1997) show that only a narrow region of the massradius plane of GCs is stable against disruption, and suggest that the initial parameter distribution may have been much larger than what is observed today. This may be what we are seeing in Fig. 8 where the young clusters occupy a much larger region of $\kappa$-space (in terms of mass, radius, and compactness) than their older globular cluster counterparts.

We note that many young star clusters are not expected to survive for more than $\sim 100$ Myr (e.g. Bastian et al. 2005a; Fall et al. 2005) due to internal and external disruption mechanisms. Thus, it can be expected that many of the youngest YMCs in our sample will never survive to comparable ages as the Galactic GCs. We do not wish to imply in Fig. 8 that all the YMCs will survive to comparable ages, only that once differences in their stellar populations are taken into account, the YMCs occupy a much larger parameter space than their old GC counterparts. In particular we note that YMCs tend to display extended envelopes in contrast to the tidally truncated older GCs (e.g. Schweizer 2004). The loss of such extended envelopes (i.e. due to interaction with their environment) may significantly change the position of the young clusters in Fig. 8. As NGC 1316:G114 is 3 Gyr old it is likely that it has already lost its extended envelope, which may explain why it falls on the relation for old GCs in Fig. 8.

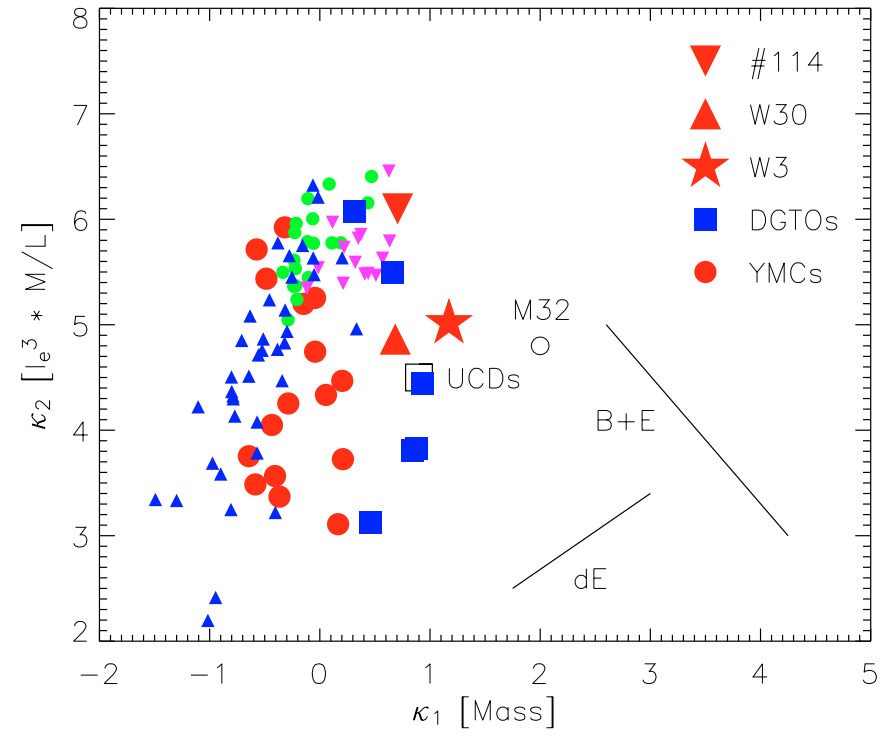

Fig. 8. $\kappa$-space: the same as Fig. 7 except that all of the YMCs have been aged to 10 Gyr using SSP models. Again we have not included any dynamical evolution of the cluster (e.g. changes in radius or velocity dispersion). Note that many of the YMCs will move into a similar region as $\mathrm{W} 3, \mathrm{~W} 30$, the UCDs, and the DGTOs once differences in the stellar populations are taken into account. The YMCs occupy a much broader region of $\kappa$-space than the old globular clusters. In this figure we do not show the evolution of the Nuclear clusters as the assumption of passive stellar evolution with no additional star formation is clearly not valid (e.g. Bõker et al. 2004).

The similarities between young massive clusters and old globular clusters have been shown in a number of recent works (e.g. Kissler-Patig 2004; de Grijs et al. 2005). In particular, Kissler-Patig (2004) showed that YMCs will follow a very similar $M_{V}-\sigma$ relation (which is one projection of $\kappa$-space) as old GCs once the fading of the young clusters (due to stellar evolution) is taken into account. However, the dangers of using such a projection can be seen when comparing Fig. 1 in Kissler-Patig (2004) with Figs. 7 and 8 in the present work. In the $M_{V}-\sigma$ projection, the UCDs appear to be quite consistent with the distribution of young and old clusters. However, in $\kappa$ space (which also includes information on the size) we see that the UCDs are disjoint from the globular clusters. Additionally, as stated above, in $\kappa$-space the young clusters clearly occupy a much larger parameter space than the old GCs, contrary to what is seen in the $M_{V}-\sigma$ projection. Finally, we note that the UCDs, DGTOs, and YMCs with masses above a few $\times 10^{6} M_{\odot}$ all appear very similar with respect to their scaling relations (i.e. mass, velocity dispersion, size, and mean mass density), suggesting a common formation mechanism (Kissler-Patig et al. 2005).

\section{Conclusions}

We have presented velocity dispersion, effective radius, and hence dynamical mass measurements of two extremely massive clusters in galactic merger remnants. These results confirm that galactic mergers can produce star clusters with masses well in excess of the most massive globular clusters in the Milky Way. 
However, we have also shown that while these clusters are extremely massive, they are consistent with being the high-mass end of a continuous power-law distribution of star clusters, suggesting that cluster formation is (mass) scale independent.

Comparing the light-to-mass ratios of W30 (in NGC 7252) and G114 (in NGC 1316) to those predicted by simple stellar population models (at the ages of the clusters), shows that both of these clusters are consistent with having Kroupa-type stellar mass functions. Applying the same analysis to other young clusters taken from the literature shows that there is a significant age dependence on how well SSP models fit the light-tomass ratios of young clusters. Therefore, it is possible that the deviation from the light-to-mass ratio of young clusters from that predicted by SSP models is not due to a varying stellar mass function, but instead reflects the state of equilibrium of the youngest clusters.

We have shown that $\mathrm{W} 30$ and G114 currently reside at the high-mass tip of the old globular cluster distribution in $\kappa$-space (a re-definition of the fundamental plane). Both clusters, along with many young clusters taken from the literature, are likely to evolve into the space occupied by the so-called ultra-compact dwarf galaxies (UCDs) and the dwarf galaxy transition objects (DGTOs). This shows that young massive clusters and UCDs/DGTOs share many similar properties and suggests that the enigmatic UCDs/DGTOs may have formed in a similar manner as the most massive globular clusters in mergers, i.e., under rather violent circumstances.

Additionally, we showed that young massive star clusters will occupy a much larger region of $\kappa$-space than presently occupied by old globular clusters. This is consistent with the interpretation that star clusters are born with a larger range of parameters (e.g. radius, mass, and compactness) than displayed by globular clusters, and destructive processes whittle away at the initial full distribution with only clusters which have parameters within a small range surviving to old ages.

Acknowledgements. We thank Marcelo Mora for his help in generating the ACS WFC PSF. Dean McLaughlin is gratefully acknowledged for providing a uniform electronic table of the parameters of the globular clusters in the Milky Way, M31, and NGC 5128. We also thank Mark Gieles for insightful discussions, as well as Linda Smith for her help in understanding the properties of cluster M82F. F.S. gratefully acknowledges partial support from the National Science Foundation through grant AST-0205994.

\section{References}

Anders, P., de Grijs, R., Fritze-v. Alvensleben, U., \& Bissantz, N. 2004, MNRAS, 347, 17

Bastian, N., Gieles, M., Lamers, H. J. G. L. M., Scheepmaker, R. A., \& de Grijs, R. 2005a, A\&A 431, 905

Bastian, N., Hempel, M., Kissler-Patig, M., Homeier, N., \& Trancho, G. 2005b, A\&A, 435, 65

Bender, R. 1990, A\&A, 229, 441

Bender, R., Burstein, D., \& Faber, S. M. 1992, ApJ, 399, 462

Bender, R., Saglia, R. P., \& Gerhard, O. 1994, MNRAS, 269, 785

Boily, C. M., \& Kroupa, P. 2003, MNRAS, 338, 665

Boily, C. M., Lançon, A., Deiters, S., \& Heggie, D. C. 2005, ApJ, 620, L27

Böker, T., Sarzi, M., McLaughlin, D. E., et al. 2004, AJ, 127, 105
Boutloukos, S. G., \& Lamers, H. J. G. L. M. 2003, MNRAS, 338, 717 Burstein, D., Bender, R., Faber, S., \& Nolthenius, R. 1997, AJ, 114, 1365

Clark, J. S., Negueruela, I., Crowther, P. A., \& Goodwin, S. P. 2005, A\&A, 434, 949

de Grijs, R., Wilkinson, M. I., \& Tadhunter, C. N. 2005, MNRAS, 361, 311

Drinkwater, M. J., Gregg, M. D., Hilker, M., et al. 2003, Nature, 423, 519

Fall, S. M., \& Rees, M. J. 1977, MNRAS, 181, 37

Fall, S. M., Chandar, R., \& Whitmore, B. C. 2005, ApJ, 631, L133

Gnedin, O. Y., \& Ostriker, J. P. 1997, ApJ, 474, 223

Goudfrooij, P., Mack, J., Kissler-Patig, M., Meylan, G., \& Minniti, D. 2001a, MNRAS, 322, 643

Goudfrooij, P., Alonso, M. V., Maraston, C., \& Minniti, D. 2001b, MNRAS, 328, 237

Goudfrooij, P., Gilmore, D., Whitmore, B. C., \& Schweizer, F. 2004, ApJ, 613, 121

Haşegan, M., Jordán, A., Côté, P., et al. 2005, ApJ, 627, 203

Hilker, M., Infante, L., Vieira, G., Kissler-Patig, M., \& Richtler, T. 1999, A\&AS, 134, 75

Ho, L. C., \& Filippenko, A. V. 1996a, ApJ, 466, L83

Ho, L. C., \& Filippenko, A. V. 1996b, ApJ, 472, 600

Holtzman, J. A., Faber, S. M., Shaya, E. J., et al. 1992, AJ, 103, 691

Kissler-Patig, M. 2004, in The Formation and Evolution of Massive Young Star Clusters (ASP), ed. H. J. G. L. M. Lamers, L. J. Smith, \& A. Nota, ASP Conf. Ser., 322, 535

Kissler-Patig, M., Jordán, A., \& Bastian, N. 2005, A\&A, submitted

Krist, J., \& Hook, R. 1997, The Tiny Tim User's Guide, STScI

Kroupa, P. 2002, Science, 295, 82

Lamers, H. J. G. L. M., Gieles, M., Bastian, N., et al. 2005, A\&A, 441, 117

Larsen, S. S. 1999, A\&AS, 139, 393

Larsen, S. S. 2004, in Planets to Cosmology: Essential Science in Hubble's Final Years, ed. M. Livio [arXiv: astro-ph/0408201]

Larsen, S. S., Brodie, J. P., \& Hunter, D. A. 2004, AJ, 128, 2295

Larsen, S. S., \& Richtler, T. 2004, A\&A, 427, 495

Maraston, C. 2005, MNRAS, 362, 799

Maraston, C., Kissler-Patig, M., Brodie, J., et al. 2001, A\&A, 370, 176

Maraston, C., Bastian, N., Saglia, R. P., et al. 2004, A\&A, 416, 467

Martini, P., \& Ho, L. C. 2004, ApJ, 610, 233

McLaughlin, D. E., \& van der Marel, R. P. 2005, ApJS, 161, 304

McCrady, N., Gilbert, A. M., \& Graham, J. R. 2003, ApJ, 596, 240

McCrady, N., Graham, J. R., \& Vacca, W. D. 2005, ApJ, 621, 278

Mengel, S., Lehnert, M. D., Thatte, N., \& Genzel, R. 2002, ApJ, 550, 280

Meylan, G., \& Mayor, M. 1986, A\&A, 166, 122

Miller, B. W., Whitmore, B. C., Schweizer, F., \& Fall, S. M. 1997, AJ, 114,2381

Salpeter, E. E. 1955, ApJ, 121, 161

Schweizer, F. 1987, in Nearly normal galaxies: From the Planck time to the present; Proc. of the Eighth Santa Cruz Summer Workshop in Astronomy and Astrophysics (New York: Springer-Verlag), 18

Schweizer, F. 2004, in The Formation and Evolution of Massive Young Star Clusters (ASP), ed. H. J. G. L. M. Lamers, L. J. Smith, \& A. Nota, ASP Conf. Ser., 322, 111

Schweizer, F., \& Seitzer, P. 1998, AJ, 116, 2206

Smith, L. J., \& Gallagher, J. S. 2001, MNRAS, 326, 1027

Spitzer, L. 1987, Dynamical Evolution of Globular Clusters, Princeton Series in Astrophysics (Princeton University Press)

Walcher, C. J., van der Marel, R. P., McLaughlin, D., et al. 2005, ApJ, 618,237

Whitmore, B. C. 2003, in A Decade of Hubble Space Telescope Science, STScI Symposium Series 14, ed. M. Livio, K. Noll, \& M. Stiavelli (Cambridge University Press), 153 